\title{
Risk Factors for Recurrent Ventricular Arrhythmias in Patients with Idiopathic Ventricular Fibrillation
}

\author{
Rand Ibrahim ${ }^{1}$ and Marwan Refaat ${ }^{1}$ \\ ${ }^{1}$ American University of Beirut Medical Center
}

July 25, 2020

Sudden Cardiac Death (SCD) remains a global threat. ${ }^{1}$ The most common causes of SCD are ischemic heart diseases and structural cardiomyopathies in the elderly. Additional causes can be arrhythmogenic, respiratory, metabolic, or even toxigenic. ${ }^{2,3,4}$ Despite the novel diagnostic tools and our deeper understanding of pathologies and genetic associations, there remains a subset of patients for whom a trigger is not identifiable. When associated with a pattern of Ventricular Fibrillation, the diagnosis of exclusion is deemed Idiopathic Ventricular Fibrillation (IVF). ${ }^{2,5}$ IVF accounts for $5 \%$ of all SCDs ${ }^{6}$ - and up to $23 \%$ in the young male subgroup ${ }^{5}$ - and has a high range of recurrence rates (11-45\%). ${ }^{7,8,9}$ There are still knowledge gaps in the initial assessment, follow-up approach, risk stratification and subsequent management for IVF. 1,10,11 While subsets of IVF presentations have been better characterized into channelopathies, such as Brugada's syndrome (BrS), Long QT Syndrome (LQTS), Early Repolarization Syndrome (ERS), Catecholaminergic Polymorphic Ventricular Tachycardia (CPVT), much remains to be discovered. ${ }^{12,13}$ Implantable Cardioverter Device (ICD) placement as secondary prevention for IVF is the standard of care. This is warranted in the setting of high recurrence rates of arrhythmias (11-43\%). Multiple studies have shown potential complications from ICDs and a significant number of cases experiencing inappropriate shock after ICD placement. ${ }^{14}$

In their article, Stampe et al. aim to further understand clinical presentation and assessment, and risk factors for recurrent ventricular arrhythmias in IVF patients. Using a single-centered retrospective study, they followed a total of 84 Danish patients who were initially diagnosed with IVF and received a secondary ICD placement between December 2007 and June 2019. Median follow-up time was 5.2 years (ICR=2-7.6). To ensure detection of many possible underlying etiologies ranging from structural, ischemic, arrhythmogenic, metabolic, or toxicologic, the researchers found that a wide array of diagnostic tools were necessary: standard electrocardiograms (ECGs), high-precordial leads ECGs, standing ECGs, Holter monitoring, sodium-channel blocker provocation tests, exercise stress tests, echocardiograms, cardiac magnetic resonance imaging, coronary angiograms, cardiac computed tomography, electrophysiological studies, histological assessment, blood tests, toxicology screens, and genetic analysis.

The study by Stampe et al. highlights the importance of thorough and continuous follow-up with rigorous evaluation: Three (3.6\%) patients initially diagnosed with IVF were later found to have underlying cardiac abnormalities (LQTS and Dilated Cardiomyopathy) that explained their SCA. Like other studies, the burden of arrhythmia was found to be high, but unlike reported data, the overall prognosis of IVF was good. Despite the initial pattern of ventricular fibrillation in those who experienced appropriate ICD placement (29.6\%), ventricular tachycardia and ventricular fibrillation had a comparable predominance. As for patients with inappropriate ICD placements, atrial fibrillation was a commonly identified pathological rhythm (16.7\%). Recurrent cardiac arrest at presentation (19.8\%) was a risk factor for appropriate ICD therapy (HR=2.63, $\mathrm{CI}=1.08-6.40, \mathrm{p}=0.033)$. However, in contrast to previous studies, early repolarization detected on baseline ECG (12.5\%), was not found to be a risk factor $(\mathrm{p}=0.842)$.

The study by Stampe et al. has few limitations. First, the study design, a retrospective cohort, precluded 
standardized follow-up frequencies and diagnostic testing. Second, while the study was included many of the cofounders tested in previous studies (baseline characteristics, baseline ECG patterns, comorbidities), medication use was not included. Third, the follow-up period may have been insufficient to detect effect from some of the confounding factors. Finally, the sample size was small and it was from a single center.

There are several strengths of the Stampe et al. study. Firstly, the wide range of diagnostic tests used at index presentation and during the follow-up period ensured meticulous detection of most underlying etiologies. Secondly, appropriate and well-defined inclusion and exclusion criteria were used. Thirdly, funding by independent parties ensured no influence on study design, result evaluation, and interpretation. Finally, the study has succeeded in improving our understanding of IVF. Future studies should include though a larger population size and a more diverse population.

References:

1.AlJaroudi WA, Refaat MM, Habib RH, Al-Shaar L, Singh M, et al. Effect of Angiotensin Converting Enzyme Inhibitors and Receptor Blockers on Appropriate Implantable Cardiac Defibrillator Shock: Insights from the GRADE Multicenter Registry. Am J Cardiol Apr 2015; 115 (7): 115(7):924-31.

2. Al-Khatib SM, Stevenson WG, Ackerman MJ, et al. 2017 AHA/ACC/HRS guideline for management of patients with ventricular arrhythmias and the prevention of sudden cardiac death: executive summary. J Am Coll Cardiol 2018;72:e91-e220.

3. Refaat MM, Hotait M, London B: Genetics of Sudden Cardiac Death. Curr Cardiol Rep Jul 2015; 17(7): 606

4. Priori SG, Wilde AA, Horie M, Cho Y, Behr ER, Berul C, et al. HRS/EHRA/APHRS expert consensus statement on the diagnosis and management of patients with inherited primary arrhythmia syndromes: document endorsed by HRS, EHRA, and APHRS in May 2013 and by ACCF, AHA, PACES, and AEPC in June 2013. Heart Rhythm 2013;10:1932-1963.

5. Priori SG, Blomström-Lundqvist C, Mazzanti A, et al. ESC Guidelines for the management of patients with ventricular arrhythmias and the prevention of sudden cardiac death: The Task Force for the Management of Patients with Ventricular Arrhythmias and the Prevention of Sudden Cardiac Death of the European Society of Cardiology (ESC). Endorsed by: Association for European Paediatric and Congenital Cardiology (AEPC). Eur Heart J 2015;36(41):2793-2867.

6. Zipes DP, Wellens HJ. Sudden cardiac death. Circulation. 1998;98:2334-2351.

7. Ozaydin M, Moazzami K, Kalantarian S, Lee H, Mansour M, Ruskin JN. Long-term outcome of patients with idiopathic ventricular fibrillation: a meta-analysis. J Cardiovasc Electrophysiol 2015;26:1095-1104.

8. Herman AR, Cheung C, Gerull B, Simpson CS, Birnie DH, Klein GJ, et al. Outcome of apparently unexplained cardiac arrest: results from investigation and follow-up of the prospective cardiac arrest survivors with preserved ejection fraction registry. Circ Arrhythm Electrophysiol 2016;9:e003619.

9. Siebermair J, Sinner MF, Beckmann BM, Laubender RP, Martens E, Sattler S, et al.Early repolarization pattern is the strongest predictor of arrhythmia recurrence in patients with idiopathic ventricular fibrillation: results from a single centre long-term follow-up over 20 years. Europace 2016;18:718-25.

10. Refaat MM, Hotait M, Tseng ZH: Utility of the Exercise Electrocardiogram Testing in Sudden Cardiac Death Risk Stratification. Ann Noninvasive Electrocardiol 2014; 19(4): 311-318.

11. Gray B, Ackerman MJ, Semsarian C, Behr ER. Evaluation after sudden death in the young: a global approach. Circ Arrhythm Electrophysiol 2019;12: e007453.

12. Herman AR, Cheung C, Gerull B, Simpson CS, Birnie DH, Klein GJ, et al. Response to Letter Regarding Article, Outcome of apparently unexplained cardiac arrest: results from investigation and follow-up of the 
prospective cardiac arrest survivors with preserved ejection fraction registry". Circ Arrhythm Electrophysiol 2016;9:e004012.

13. Chen Q, Kirsch GE, Zhang D, Brugada R, Brugada J, Brugada P, Potenza D, et al. Genetic basis and molecular mechanism for idiopathic ventricular fibrillation. Nature 1998;392:293-296.

14. Baranchuk A, Refaat M, Patton KK, Chung M, Krishnan K, et al. What Should You Know About Cybersecurity For Cardiac Implantable Electronic Devices? ACC EP Council Perspective. J Am Coll Cardiol Mar 2018; 71(11):1284-1288. 